\title{
Controle glicêmico em pacientes críticos na UTI
}

\author{
Glycemic control in critically ill patients in the ICU
}

WiLLIAM O. Silva*

\section{Resumo}

O uso do controle glicêmico estrito (glicemia entre $80-110 \mathrm{mg} / \mathrm{dl}$ ) em pacientes críticos foi alvo de numerosos trabalhos com resultados divergentes. A partir de resultados expressivos observados no estudo de Leuven $\mathrm{I},{ }^{2}$ em que se utilizou um controle estrito de glicemia com redução da mortalidade, este tipo de manuseio tornou-se rotina nas UTIs. Entretanto, com os resultados dos trabalhos mais recentes, houve uma rediscussão do tema, objetivando orientar os intensivistas a respeito do nível de controle glicêmico ideal nestes pacientes. Sabe-se que a hiperglicemia é deletéria no paciente crítico nos vários cenários clínicos encontrados, implicando em desfechos piores. Contudo, a hipoglicemia que ocorre com maior frequência quando se estabelece um protocolo estrito, impacta de forma negativa os resultados quando tentamos normalizar a glicemia de pacientes criticamente enfermos. Constitui um grande desafio a manutenção da glicemia em valores aceitáveis neste grupo de pacientes com grandes alterações metabólicas e neste sentido a instituição de um protocolo de controle glicêmico é fundamental. Estudos demostram que a hiperglicemia está associada à disfunção imunológica e endotelial, assim como a alterações de coagulação e distúrbios hidroeletrolíticos. Recente guideline publicado pelo Surviving Sepsis Campaign reenfatiza o papel do controle moderado da glicemia em paciente sépticos (igual ou abaixo de $180 \mathrm{mg} /$ dl). Na nossa unidade o objetivo é manter a glicemia ente $140-180 \mathrm{mg} / \mathrm{dl}$ nos pacientes com resposta inflamatória sistêmica. Aguardam-se novos estudos que determinem o valor ideal de glicemia nestes pacientes, assim como o melhor meio de alcançá-lo, seja pelo uso de algoritmos computorizados e/ou controle de glicemia por glucômetros de leitura contínua transdérmicos.

Descritores: Hiperglicemia; Hipoglicemia; Controle; Estado terminal; Mortalidade; Comportamento de redução do risco; Unidades de terapia intensiva. 


\section{Abstract}

The use of strict glycemic control (blood glucose between $80-110 \mathrm{mg} / \mathrm{l}$ ) in critically ill patients has been the subject of numerous studies with conflicting results. From impressive results observed in Leuven I study, ${ }^{2}$ which used a strict control of glycemia with mortality reduction, this type of management has become routine in the ICU. However, with the results of more recent works, there has been a renewed discussion of the subject, aiming to guide intensivists regarding the optimal level of glycemic control in these patients. It is known that hyperglycemia is deleterious in critically ill patients in various clinical scenarios encountered in ICU, resulting in worse outcomes. However, hypoglycemia which occurs most commonly when establishing a strict protocol, negatively affects the results when we try to normalize glycemia of critically ill patients. It is a major challenge to maintain acceptable blood glucose values in this group of patients with major metabolic derangements and in this regard the establishment of a protocol for glycemic control is critical. Studies show that hyperglycemia is associated with immune and endothelial dysfunction as well as alterations in coagulation and fluids and electrolyte disorders. In our unit the goal is to keep blood glucose levels being 140-180 $\mathrm{mg} / \mathrm{dl}$ in patients with systemic inflammatory response. Recent guideline of the Surviving Sepsis Campaign reemphasize de loose glycemic control (less ou equal than $180 \mathrm{mg} / \mathrm{gl}$ ) in septic patients. Further studies are awaited to determine the optimal blood glucose in these patients, as well as the best means of reaching it, either by the use of computerized algorithms and/or glycemic control by continuous transdermal glucometers reading.

Keywords: Hyperglycemia; Hypoglycemia; Control; Critical illness; Mortality; Risk reduction Behavior; Intensive care units.

\section{Introdução}

A hiperglicemia é uma resposta metabólica comum em pacientes gravemente enfermos não diabéticos. Durante muitos anos foi considerada somente uma resposta fisiológica ao trauma ou infecção cuja magnitude se correlacionava com a gravidade da patologia subjacente. ${ }^{1,2}$ Entretanto, vários estudos claramente demostraram uma associação entre a hiperglicemia e a mortalidade em várias condições clínicas críticas como infarto agudo do miocárdio, trauma, cirurgia cardíaca e sepse. ${ }^{3,45,6}$ Partindo deste pressuposto, um estudo clínico em Leuven (Bélgica), em 2001, estabeleceu um protocolo de controle glicêmico estrito (Tabela 1) em uma unidade de terapia intensiva (UTI) de pacientes cirúrgicos, relatando uma redução significativa na mortalidade, $4 \%$ de redução na mortalidade absoluta, quando o controle glicêmico era obtido (glicemias entre 80-110 mg) comparado com o controle convencional da glicemia. ${ }^{2}$ Observou-se também uma redução na incidência de insuficiência renal, polineuropatia dos pacientes críticos, taxa de infecção, hiperbilirrubinemia, dias em ventilação mecânica e dias de internação na UTI. A partir de então se estabeleceu uma mudança de paradigma e vários trabalhos uni e multicêntricos $^{7-12}$ tentaram demostrar o benefício deste tratamento em pacientes clínicos críticos, sem, entretanto, obter o mesmo resultado e suscitando dúvidas sobre o mecanismo da redução de mortalidade obtida no primeiro trabalho. Houve até mesmo um pequeno aumento da mortalidade dos pacientes submetidos a esta terapia, provavelmente em função do aumento da incidência de hipoglicemia, definida como glicemia menor que $40 \mathrm{mg} / \mathrm{dl}^{11,13}$

Sabe-se que a introdução de um protocolo de controle glicêmico estrito é de difícil execução pela complexidade e necessidade do envolvimento de toda a equipe multidisciplinar. ${ }^{14}$ Variabilidades como a forma de coleta de sangue da glicemia, a fonte da coleta, ${ }^{15}$ a frequência da coleta, o tempo necessário para obter o controle glicêmico e a variação dos níveis glicêmicos podem influenciar no resultado final do protocolo. ${ }^{16}$ Devemos considerar também que a incidência de hipoglicemia aumenta com 
Tabela 1. Estudos controlados e randomizados de controle glicêmico estrito

\begin{tabular}{|c|c|c|c|c|c|c|c|}
\hline Estudo & $\begin{array}{c}\text { van den } \\
\text { Berghe et al. } \\
\text { Leuven I }\end{array}$ & $\begin{array}{c}\text { van den } \\
\text { Berghe et al. } \\
\text { Leuven II }\end{array}$ & $\begin{array}{c}\text { de la Rosa } \\
\text { et al. }\end{array}$ & Arabi et al. & $\begin{array}{c}\text { Brunkhorst } \\
\text { at al. }\end{array}$ & $\begin{array}{l}\text { Finfer et al. } \\
\text { NICE-SUGAR }\end{array}$ & Preiser et al. \\
\hline Ano & 2001 & 2006 & 2008 & 2008 & 2008 & 2009 & 2009 \\
\hline $\begin{array}{l}\text { Glicose } \\
\text { estrito }\end{array}$ & $80-110$ & $80-110$ & $80-110$ & $80-110$ & $80-110$ & 81-108 & $80-110$ \\
\hline $\begin{array}{c}\text { Glicose } \\
\text { convencional }\end{array}$ & $180-200$ & $180-200$ & $180-200$ & $180-200$ & $180-200$ & $144-180$ & $140-180$ \\
\hline $\begin{array}{l}\text { Mortalidade } \\
\text { estrito }\end{array}$ & 4,6 & 43 & 36 & 13,5 & 25 & 22 & 17,2 \\
\hline $\begin{array}{l}\text { Mortalidade } \\
\text { convencional }\end{array}$ & 8,0 & 52,2 & 32,4 & 17 & 26 & 21 & 15,3 \\
\hline
\end{tabular}

a adoção de controle estrito e sua identificação pelo quadro clínico em pacientes críticos é prejudicada, resultando em neuroglicopenia e contribuindo para morbidade e mortalidade. ${ }^{17}$ Embasada em vários estudos controlados e randomizados, a recomendação da Associação Americana de Diabetes (ADA) e da Associação Americana de Endocrinologistas Clínicos é um controle glicêmico moderado com glicemia entre $140-180 \mathrm{mg} / \mathrm{dl}$.

\section{Regulação da glicose no paciente crítico}

A hiperglicemia é uma alteração comum em pacientes críticos e é atribuída à resposta fisiológica ao trauma. Os níveis glicêmicos são mantidos de forma fisiológica pela interação entre a secreção de insulina, captação celular da glicose (glicólise e glucogeniogênese), produção hepática de glicose (glicogenólise e gluconeogênese) e absorção intestinal. O aumento da glicemia está em acordo com o aumento das demandas metabólicas nestes pacientes e na maioria dos casos é acompanhada de um aumento da secreção de insulina. $\mathrm{O}$ transporte da glicose para as células é facilitado por uma série de transportadores, GLUT 1 a 12, sendo o GLUT 4 o principal transportador de glicose para tecidos como o adiposo, o muscular e o cardíaco. A presença de insulina é fundamental para a função do GLUT 4. Tecidos como endotélio, epitélio, sistema nervoso central, hepatócitos e células inflamatórias não necessitam de insulina para transporte de glicose intracelular, fazendo-o através de transportadores não insulinodependentes, como GLUT 1, 2 e 3. A glicemia nos pacientes críticos encontra-se aumentada por aumento endógeno da produção hepática, mas também pode ser gerada por causas como aporte glicídico aumentado em dietas enterais e parenterais, soluções dialíticas e uso de glicocorticoide e substâncias vasopressoras. ${ }^{18} \mathrm{O}$ aumento da produção de causa endógena se faz através da liberação de hormônios contra regulatórios como glucagon, epinefrina, hormônio do crescimento e cortisol que estimulam a gluconeogênese hepática. A 
Resposta inflamatória
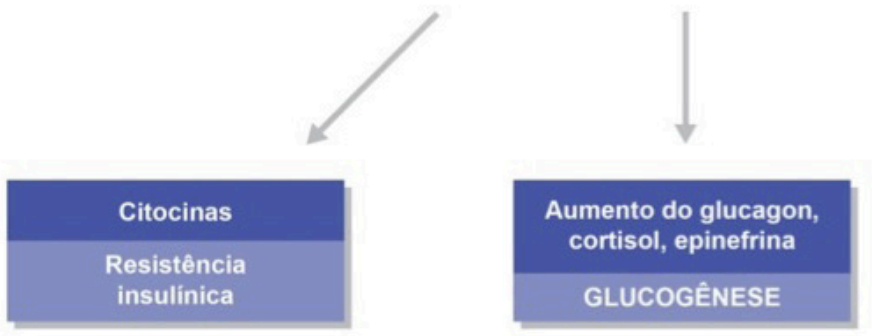

\section{Figura 1. Mecanismo dos efeitos adversos da hiperglicemia em pacientes críticos}

liberação de citocinas pró-inflamatórias como interleucina 6 e fator de necrose tumoral alfa limita a resposta periférica de tecidos dependentes de insulina promovendo, assim, uma resistência a sua ação. ${ }^{19}$ Em algum momento este processo fisiológico se torna não adaptativo, promovendo manutenção de níveis elevados de glicose nos pacientes críticos. ${ }^{20}$

\section{Efeitos da hiperglicemia nos pacientes críticos}

Além de promover seus efeitos sistêmicos osmóticos e afetar o balanço hídrico dos pacientes, a hiperglicemia aguda, definida como glicemia aleatória maior que $200 \mathrm{mg} /$ dl, é capaz de alterar a resposta inflamatória e imune, alterando também a adesão de células inflamatórias, quimiotaxia e fagocitose..$^{20} \mathrm{Em}$ condições habituais a hiperglicemia aguda infrarregula a expressão dos receptores GLUT e assim impede a entrada excessiva de glicose nas células, entretanto foi demostrado que as citocinas pró-inflamatórias promovem suprarregulação de receptores GLUT 1 em tecidos não dependentes da ação da insulina. O excesso de glicose intracelular promove, através da fosforilação oxidativa, a geração de radicais livres que promovem dano celular. Outros fatores associados à hiperglicemia como aumento da permeabilidade vascular, angiogênese e oclusão capilar levam à piora de lesão de órgãos-alvo ${ }^{18}$ 
(Figura 1). Neste contexto, a injúria celular induzida pela hiperglicemia poderia ser evitada e, portanto, a incidência de lesão de órgãos-alvo em pacientes sépticos, principalmente, poderia ser reduzida com adoção de protocolos de tratamento da hiperglicemia aguda. Confirmando estes dados, Krinsley e colaboradores reportaram uma associação entre os níveis glicêmicos e mortalidade em pacientes críticos, com mortalidade de $9,6 \%$ quando a glicemia foi mantida entre 80 e $99 \mathrm{mg} / \mathrm{dl}$ e $42,5 \%$ quando a glicemia era superior a $300 \mathrm{mg} / \mathrm{dl} .{ }^{5}$ Também foi demostrado que em paciente com infarto agudo do miocárdio, a hiperglicemia eleva o risco de insuficiência cardíaca, choque cardiogênico e mortalidade hospitalar. ${ }^{21}$ Em pacientes com trauma cranioencefálico a hiperglicemia se associa a um maior tempo de internação, pior prognóstico funcional e maior mortalidade, ${ }^{22}$ assim como no paciente com acidente vascular cerebral há uma piora da recuperação funcional e maior mortalidade. ${ }^{23}$

\section{Efeitos da insulina nos pacientes críticos}

Além do óbvio efeito metabólico de controle da glicose a infusão de insulina age antagonizando uma série de efeitos deletérios descritos acima, e promove melhor regulação dos distúrbios envolvendo os lipídeos, aumenta o anabolismo, reduz a geração de citocinas inflamatórias, previne a disfunção endotelial e reequilibra o status do sistema de coagulação no paciente crítico. ${ }^{20}$

\section{Estudos de controle glicêmico estrito}

Partindo do pressuposto que a hiperglicemia, apesar de ser uma resposta fisiológica ao estresse no paciente crítico, estava associada a alterações funcionais que promoveriam uma maior mortalidade, foram conduzidos vários trabalhos sobre uso intensivo de infusões venosas de insulina regular com objetivo de manter a glicemia em níveis estritos de 80-110 $\mathrm{mg} / \mathrm{dl}$, sendo o primeiro deles conduzido em Leuven, na Bélgica, no ano de 2001. ${ }^{2}$ Em uma unidade primordialmente de pacientes em pósoperatório de cirurgia cardíaca, comparou-se um protocolo de insulinoterapia com objetivo de níveis glicêmicos entre 80 e 110 mg/dl com um grupo-controle no qual a insulinoterapia se iniciava com glicemia acima de $215 \mathrm{mg} / \mathrm{dl}$ e depois era mantida em níveis de 180-200 mg/ dl. A mortalidade foi significativamente mais baixa no grupo estrito com taxa de mortalidade de $4,6 \%$ comparada com $8 \%$ no controle. Além disso, observaram-se menos $41 \%$ de necessidade de diálise, $46 \%$ menos de sepse, $44 \%$ menos de polineuropatia e $50 \%$ menos de necessidade de transfusões sanguíneas. Estes resultados surpreendentes levaram outros grupos e o mesmo grupo de Leuven a realizar estudos semelhantes e a tentarem reproduzir estes dados em uma população mista de pacientes clínicos e cirúrgicos. Estudos conduzidos por De la Rosa e colaboradores, ${ }^{9}$ Arabi e colaboradores, ${ }^{8}$ Brunkhorst e colaboradores, ${ }^{10}$ este interrompido precocemente pela incidência de hipoglicemia, $\mathrm{e}$ Preiser e colaboradores ${ }^{12}$ não relatam diferenças estatísticas entre os dois grupos. Em 2006 Van den Berghe colaboradores ${ }^{7}$ (Leuven II) também não conseguiram reproduzir os dados do primeiro estudo, mas mostraram um menor tempo de internação hospitalar, tempo em ventilação mecânica e necessidade de diálise no grupo de controle estrito com permanência na UTI maior que três dias.

O maior estudo comparativo entre controle glicêmico estrito e convencional (The Normoglycemia in Intensive Care Evaluation - Survival Using Glucose Algorithm Regulation Study - NICE SUGAR) foi realizado em 2009 por Finfer e colaboradores, ${ }^{11}$ contabilizando 6.104 pacientes, em múltiplos centros. Além de não ter demostrado diferença de mortalidade entre os grupos, houve na realidade um aumento da mortalidade no grupo de controle restrito. Estes resultados muito diferentes entre os dois principais trabalhos comparativos (Leuven I e NICE-SUGAR) revelaram também, a despeito 
do não beneficio do controle estrito, diferenças metodológicas entre estes trabalhos. No primeiro estudo, de Van den Berghe e colaboradores, a maioria dos pacientes era de pós-operatório de cirurgia cardíaca, que diferem de pacientes com sepse grave ou traumas extensos em termos de resposta metabólica ao trauma. A maior parte destes pacientes foi submetida à reposição precoce de calorias que podem ter influenciado na baixa incidência de hipoglicemia. Todos os pacientes do estudo de Leuven I foram controlados rigidamente com glicemia arterial, e tanto a velocidade de se obter um controle glicêmico adequado quanto os níveis médios de glicemia foram mais baixos do que no NICE-SUGAR. O grupo-controle dos pacientes de Leuven I tinham níveis glicêmicos muito elevados, não justificados pelo atual nível de conhecimento, o que pode ter levado a diferenças importantes no desfecho. O trabalho do grupo de Finfer e colaboradores, entretanto, reflete mais a realidade de nossos serviços de terapia intensiva, com pacientes mistos, clínicos e cirúrgicos, controle de glicemia variável, utilizando na maior parte a glicemia capilar, e a dificuldade de conseguir um rápido ajuste na glicose de pacientes muito graves. O controle do protocolo de glicemia também pode ter influenciado a incidência de hipoglicemia, assim como o fato de a glicemia ter sido realizada por aparelhos de gasometria no estudo de Leuven pode ter antecipado o diagnóstico coadjuvante de hipocalemia, alteração hidroeletrolítica comum com infusões de insulina em doses elevadas. Como curiosidade, este fato pode ter sido responsável pelo aumento da mortalidade cardíaca observada no trabalho de Finfer e colaboradores. Estudos recentes sugerem que a variabilidade dos níveis glicêmicos, ou seja, a amplitude das alterações da glicemia, pode influenciar os desfechos de pacientes críticos submetidos a controle estrito da glicose de forma independente. ${ }^{24,25,26}$ Não há dados em nenhum destes trabalhos a este respeito, sendo, portanto, um dos fatores que podem ter influenciado os resultados do trabalho de Finfer e colaboradores em que os pacientes foram submetidos a algoritmos de infusão de insulina com variações glicêmicas maiores.

Em resumo, os níveis elevados de glicemia parecem estar relacionados à mortalidade aumentada nos pacientes críticos e não refletem meramente uma resposta metabólica ao trauma. O controle estrito da glicemia a níveis de 80-110 $\mathrm{mg} / \mathrm{dl}$ parece não beneficiar pacientes críticos e até mesmo pode estar associado a aumento da mortalidade. A hipoglicemia parece ser muito mais frequente nesse grupo e pode contribuir significativamente na mortalidade. ${ }^{26,27,17} \mathrm{Em}$ estudos de metanálise também não foi demostrado o papel deste controle glicêmico estrito na função renal ou tempo de permanência na UTI, ${ }^{28,29}$ como visto no primeiro estudo do grupo de Leuven. Recente guideline, publicado pelo Surviving Sepsis Campaign, reenfatiza o controle glicêmico igual ou abaixo de $180 \mathrm{mg} /$ $\mathrm{dl}$, não impondo limite ao controle mínimo. ${ }^{30}$

Baseados nestes fatores, instituímos na nossa UTI um protocolo de controle glicêmico (Anexo I), de fácil execução e entendimento pela equipe multidisciplinar, sob supervisão médica, objetivando o controle ótimo da glicemia entre 140-180 mg/dl, conforme recomendação da American Diabetes Association e da American Association of Clinical Endocrinologists. Enfatizamos o uso deste protocolo em todo o paciente com resposta inflamatória sistêmica, estimulando o início precoce de fonte calórica preferencialmente enteral, dando prioridade para glicemias obtidas de linha arterial e instituindo a pronta-correção da hipoglicemia.

\section{Referências}

1. Mizock BA. Blood glucose management during critical illness. Rev Endocr Metab Disord. 2003;4(2):187-94.

2. van den Berghe $G$, Wouters P, Weekers F, Verwaest C, Bruyninckx F, Schetz M, et al. Intensive insulin therapy in critically ill patients. N Engl J Med. 2001 Nov 8;345(19):1359-67.

3. Christiansen C, Toft P, Jørgensen HS, Andersen SK, Tønnesen E. Hyperglycaemia and mortality in critically ill patients. A prospective study. Intensive Care Med. 2004 Aug;30(8):1685-8. 
4. Gabbanelli V, Pantanetti S, Donati A, Principi T, Pelaia P. Correlation between hyperglycemia and mortality in a medical and surgical intensive care unit. Minerva Anestesiol. 2005 Nov;71(11):717-25.

5. Krinsley JS. Association Between Hyperglycemia and Increased Hospital Mortality in a Heterogeneous Population of Critically Ill Patients. Mayo Clin Proc. 2003;78(12):1471-8.

6. Lazzeri C, Tarquini R, Giunta F, Gensini GF. Glucose dysmetabolism and prognosis in critical illness. Intern Emerg Med. 2009 Apr;4(2):147-56. http://dx.doi.org/10.1007/ s11739-008-0206-3

7. van den Berghe G, Wouters PJ, Bouillon R, Weekers F, Verwaest C, Schetz M, et al. Outcome benefit of intensive insulin therapy in the critically ill: Insulin dose versus glycemic control. Crit Care Med. 2003 Feb;31(2):359-66.

8. Arabi YM, Dabbagh OC, Tamim HM, AlShimemeri AA, Memish ZA, Haddad SH, et al. Intensive versus conventional insulin therapy: a randomized controlled trial in medical and surgical critically ill patients. Crit Care Med. 2008 Dec;36(12):3190-7. http:// dx.doi.org/10.1097/CCM.0b013e31818f21aa

9. de la Rosa GDC, Donado JH, Restrepo AH, Quintero AM, González LG, Saldarriaga $\mathrm{NE}$, et al. Strict glycaemic control in patients hospitalised in a mixed medical and surgical intensive care unit: a randomised clinical trial. Crit Care. 2008;12(5):R120. http://dx.doi. org/10.1186/cc7017

10. Brunkhorst FM, Engel C, Bloos F, MeierHellmann A, Ragaller M, Weiler N, et al. Intensive insulin therapy and pentastarch resuscitation in severe sepsis. N Engl J Med. 2008 Jan 10;358(2):125-39. http://dx.doi. org/10.1056/NEJMoa070716

11. NICE-SUGAR Study Investigators,Finfer S, Chittock DR, Su SY-S, Blair D, Foster D, et al. Intensive versus conventional glucose control in critically ill patients. N Engl J Med. 2009 Mar 26;360(13):1283-97. http://dx.doi. org/10.1056/NEJMoa0810625

12. Preiser J-C, Devos P, Ruiz-Santana S, Mélot C, Annane D, Groeneveld J, et al. A prospective randomised multi-centre controlled trial on tight glucose control by intensive insulin therapy in adult intensive care units: the Glucontrol study. Int Care Med. 2009;35(10):1738-48. http://dx.doi.org/10.1007/ s00134-009-1585-2

13. Kavanagh BP. Glucose in the ICU- evidence, guidelines, and outcomes. N Engl J Med. 2012;367(13):1259-60.

14. Holzinger U, Feldbacher M, Bachlechner A, Kitzberger R, Fuhrmann V, Madl C. Improvement of glucose control in the intensive care unit: an interdisciplinary collaboration study. Am J Crit Care. 2008;17(2):150-6.
15. Juneja D, Pandey R, Singh O. Comparison between arterial and capillary blood glucose monitoring in patients with shock. Eur J Intern Med. 2011 Jun;22(3):241-4. http:// dx.doi.org/10.1016/j.ejim.2011.01.004

16. Taylor BE, Schallom ME, Sona CS, Buchman TG, Boyle WA, Mazuski JE, et al. Efficacy and safety of an insulin infusion protocol in a surgical ICU. J Am Coll Surg. 2006 Jan;202(1):1-9.

17. Anabtawi A, Hurst M, Titi M, Patel S, Palacio C, Rajamani K. Incidence of hypoglycemia with tight glycemic control protocols: a comparative study. Diabetes Technol Ther. 2010 Aug;12(8):635-9. http://dx.doi. org/10.1089/dia.2010.0009

18. Kovalaske MA, Gandhi GY. Glycemic control in the medical intensive care unit. J Diabetes Sci Technol. 2009;3(6):1330-41.

19. Zauner A, Nimmerrichter P, Anderwald C, Bischof M, Schiefermeier M, Ratheiser K, et al. Severity of insulin resistance in critically ill medical patients. Metabolism. 2007;56(1):1-5.

20. van den Berghe G. Science in medicine How does blood glucose control with insulin save lives in intensive care? J Clin Invest. 2004 Nov;114(9):1187-95.

21. Capes SE, Hunt D, Malmberg K, Gerstein HC. Stress hyperglycaemia and increased risk of death after myocardial infarction in patients with and without diabetes: a systematic overview. Lancet. 2000;355(9206):773-8.

22. Bochicchio GV, Sung J, Joshi M, Bochicchio K, Johnson SB, Meyer W, et al. Persistent hyperglycemia is predictive of outcome in critically ill trauma patients. J Trauma. 2005;58(5):921-4.

23. Capes SE, Hunt D, Malmberg K, Pathak $\mathrm{P}$, Gerstein HC. Stress hyperglycemia and prognosis of stroke in nondiabetic and diabetic patients: a systematic overview. Stroke. 2001;32(10):2426-32.

24. Krinsley JS. Glycemic variability: a strong independent predictor of mortality in critically ill patients. Crit Care Med. 2008;36(11):3008-13.

25. Matsushima K, Peng M, Velasco C, Schaefer E, Diaz-Arrastia R, Frankel H. Glucose variability negatively impacts long-term functional outcome in patients with traumatic brain injury. J Crit Care. 2012 ;27(2):125-31. http://dx.doi.org/10.1016/j.jcrc.2011.08.012

26. Egi M, Bellomo R, Stachowski E, French CJ, Hart GK, Taori G, et al. Hypoglycemia and outcome in critically ill patients. Mayo Clin Proc. 2010. Mar;85(3):217-24. http://dx.doi. org/10.4065/mcp.2009.0394

27. D'Ancona G, Bertuzzi F, Sacchi L, Pirone F, Stringi V, Arcadipane A, et al. Iatrogenic hypoglycemia secondary to tight glucose control is an independent determinant for mortality and cardiac morbidity. Eur J 
Cardiothorac Surg. 2011 Aug;40(2):360-6. http://dx.doi.org/10.1016/j.ejcts.2010.11.065

28. Wiener RS, Wiener DC, Larson RJ. Benefits and risks of tight glucose control in critically ill adults: a meta-analysis. JAMA. 2008;300(8):933-44. http://dx.doi.org/10.1001/ jama.300.8.933

29. Griesdale DEG, De Souza RJ, Van Dam RM, Heyland DK, Cook DJ, Malhotra A, et al. Intensive insulin therapy and mortality among critically ill patients: a meta-analysis including NICE-SUGAR study data. CAMJ. 2009;180(8):821-7. http://dx.doi.org/10.1503/ cmaj.090206

30. Dellinger RP, Levy MM, Rhodes A, Annane D, Gerlach H, Opal SM, et al. Surviving Sepsis Campaign. Crit Care Med 2013 Feb;41(2):580-637. http://dx.doi.org/10.1097/ CCM.0b013e31827e83af

30. Dellinger RP, Levy MM, Rhodes A, et al. Surviving Sepsis Campaign. Crit Care Med. 2013;41(2):580-637. 


\section{Anexo I - Protocolo de controle intensivo de glicemias}

Meta: Manter glicemia entre 140 e $180 \mathrm{mg} / \mathrm{dl}$.

Abrangência: Pacientes adultos com resposta inflamatória sistêmica e hiperglicemia, exceto aqueles com emergências diabéticas (cetoacidose ou estado hiperosmolar).

Padronização: Preparar solução 30 minutos antes da infusão ou do término da solução anterior (para trocas) visando à estabilização de solução. Desprezar $50 \mathrm{ml}$ da solução antes da instalação da infusão venosa contínua de insulina, para preenchimento do equipo e adsorção da insulina às paredes do mesmo. Este procedimento deverá ser repetido a cada troca de solução (geralmente a cada 72 horas).

Dripping: Insulina regular humana $1 \mathrm{ml}+$ soro fisiológico $0,9 \% 99 \mathrm{ml}$.

\section{Cuidados:}

- As glicemias devem ser sempre colhidas de extremidades dos dedos e orelhas, em sistema de rodízio. Caso haja coincidência com coleta de gasometria, usar essa amostra.

- As infusões de insulina devem ser instaladas em (ordem de escolha):

1. Via venosa central exclusiva;

2. Via venosa central sem NPT e sem drogas vasoativas;

3. Via venosa central com cuidado na administração de drogas em bolus.

- A troca das soluções deve ser feita:

1. A cada 24 horas OBRIGATORIAMENTE;

2. A cada troca de acesso venoso;

3. Na presença de incompatibilidade visível de soluções (precipitações).
- Atenção extra deve ser dada aos pacientes (ALTO RISCO DE HIPOGLICEMIA):

- Sob dieta zero;

- Sob hemodiálise;

(Nestes pacientes, observar DIETA ENTERAL e infusão de SORO GLICOSADO.)

- Em caso de HIPOGLICEMIA:

- Avisar imediatamente médico e enfermeiro;

- Aplicar IMEDIATAMENTE $30 \mathrm{ml}$ de glicose a $50 \%$;

- Solicitar avaliação do médico para instalação de soro glicosado.

\section{Recomendações:}

1. As glicemias devem ser checadas em TODOS os pacientes na admissão no CTI.

2. As mensurações devem ser anotadas no balanço hídrico.

3. Glicemias acima de 180 devem respeitar o esquema de insulina regular subcutânea descrito:

a. 180-220: 2 (duas) unidades;

b. 221-260: 4 (quatro) unidades;

c. 261-300: 6 (seis) unidades;

d. 300-340: 8 (oito) unidades (avisar médico).

4. Duas glicemias capilares consecutivas MAIORES que 180 (mesmo após insulina regular), E/OU uma glicemia MAIOR que 340, determinam o início da infusão venosa contínua de insulina.

5. Início da infusão venosa contínua de insulina determina, OBRIGATORIAMENTE, glicemias capilares HORÁRIAS.

6. A suspensão da infusão venosa contínua de insulina implica em mensurações HORÁRIAS da glicemia capilar pelas 6 horas seguintes, INDEPENDENTE de seu valor. Após este período a mensuração deverá ser feita a cada 2 (duas) 
horas até serem completadas 24 horas de sua paralisação.

7. Pacientes em DIETA ZERO:

a. TODOS os pacientes em dieta zero devem receber infusão de GLICOSE (com oferta de $100 \mathrm{~g}$ de glicose), INDEPENDENTE de presença de diabetes mellitus ou início de infusão venosa contínua de insulina.

b. O início infusão venosa contínua de insulina NÃO IMPLICA na suspensão da infusão de soro glicosado (apenas seu ajuste).

DUAS glicemias maiores que 180 ou UMA maior que 340:

Iniciar dripping:

\begin{tabular}{|ccc|}
\hline Glicemia & Infusão inicial & Bolus venoso \\
$181-250$ & $2 \mathrm{ml} / \mathrm{h}$ & - \\
$251-300$ & $2 \mathrm{ml} / \mathrm{h}$ & $2 \mathrm{ml}$ \\
$300-340$ & $3 \mathrm{ml} / \mathrm{h}$ & $4 \mathrm{ml}$ \\
$>341$ & $4 \mathrm{ml} / \mathrm{h}$ & $6 \mathrm{ml}$ \\
\hline
\end{tabular}

\section{Manutenção (HGT horário):}

\begin{tabular}{|c|c|}
\hline Glicemia & Conduta \\
\hline$<70$ & $\begin{array}{l}\text { - } \quad \text { Suspender infusão. } \\
\text { - } \quad \text { Checar dieta (ou soro glicosado). } \\
\text { - } \quad \text { Administrar imediatamente } 3 \text { ampolas de glicose } 50 \% \text {. }\end{array}$ \\
\hline $70-99$ & $\begin{array}{l}\text { - } \quad \text { Suspender infusão. } \\
\text { - } \quad \text { Checar de } \mathrm{h} / \mathrm{h} \text { por até } 6 \text { horas. } \\
\text { - } \quad \text { Após } 6 \text { horas, se mantiver entre } 70 \text { e } 99 \text {, checar de } 2 / 2 \mathrm{~h} \text { por } 24 \mathrm{~h} \text {. } \\
\text { - } \quad \text { Se }>140 \text { : Reiniciar dripping com infusão anterior }-1 \mathrm{ml} / \mathrm{h} \text {. }\end{array}$ \\
\hline $100-140$ & - Reduzir infusão em $50 \%$. \\
\hline $141-180$ & - $\quad$ META ATINGIDA - MANTER INFUSÃO \\
\hline$>180$ & - Seguir tabela abaixo (Glicemia). \\
\hline
\end{tabular}

\begin{tabular}{|c|c|c|}
\hline Glicemia & Infusão $<\mathbf{1 0 ~ m l} / \mathbf{h}$ & $+2 \mathrm{ml} / \mathrm{h}$ \\
\hline $181-200$ & $+1 \mathrm{ml} / \mathrm{h}$ & $+3 \mathrm{ml} / \mathrm{h}$ \\
\hline $200-240$ & $+2 \mathrm{ml} / \mathrm{h}$ & $+4 \mathrm{ml} / \mathrm{h}$ \\
\hline $240-300$ & $+3 \mathrm{ml} / \mathrm{h}$ & Bnfusão $>\mathbf{1 0} \mathrm{ml} / \mathrm{h}$ \\
\hline$>300$ & $\begin{array}{c}\text { Bolus: } 3 \mathrm{ml} \\
\mathrm{E}\end{array}$ & $\begin{array}{c}\mathrm{E} \\
\text { Bomba: }+2 \mathrm{ml} / \mathrm{h}\end{array}$ \\
\hline
\end{tabular}

